\title{
Few-body reactions investigated with the Trojan Horse Method
}

\author{
Roberta Spartá $^{1,2 \star}$, Giuseppe G. Rapisarda ${ }^{2}$, Claudio Spitaleri ${ }^{2}$, Marco La Cognata ${ }^{2}$, \\ Rosario G. Pizzone ${ }^{2}$, Stefano Romano ${ }^{1,2}$ and Aurora Tumino ${ }^{2,3}$ \\ 1 Dipartimento di Fisica e Astronomia E. Majorana, \\ Universitá degli Studi di Catania, Catania, Italy \\ 2 Laboratori Nazionali del Sud, INFN, Catania, Italy \\ 3 Facoltá di Ingegneria e Architettura, Universitá Kore, Enna, Italy \\ $\star$ rsparta@lns.infn.it
}

\section{鹖被 Surrey, UK, 2-6 September 2019 doi:10.21468/SciPostPhysProc.3}

Proceedings for the 24th edition of European Few Body Conference,

\begin{abstract}
The Trojan Horse Method is an indirect method to measure reaction cross sections at energies of interest for nuclear astrophysics, exploiting the nuclei clustering properties. Here it is presented with its general features and detailed for the case of the ${ }^{2} \mathrm{H}(\mathrm{d}, \mathrm{p})^{3} \mathrm{H}$ and ${ }^{2} \mathrm{H}(\mathrm{d}, \mathrm{n})^{3} \mathrm{He}$ measurements, where interesting results for astrophysics and energy fusion power plants have been obtained.
\end{abstract}

(c) (i) Copyright R. Spartá et al.

This work is licensed under the Creative Commons Attribution 4.0 International License.

Published by the SciPost Foundation.
Received 15-11-2019

Accepted 09-01-2020

Published 26-02-2020

doi:10.21468/SciPostPhysProc.3.033

\section{Introduction}

The Trojan Horse Method (THM) is a well established indirect technique introduced to study charged particles nuclear reactions taking place in astrophysical environments. First introduced by Baur [1], it was experimentally realized and worked out by the AsFiN group at the Laboratori Nazionali del Sud in Italy, taking advantage of the experience on quasi-free (QF) reaction mechanism developed in the previous decades in that Laboratory.

The THM allows to extract the cross section of a two-body process $x+a \rightarrow b+B$, at very low energy ( $E_{c m}$ down to zero $\mathrm{MeV}$ ) performing the measurement of a suitable three-body reaction $a+A \rightarrow b+B+s$ in the quasi-free kinematic regime. Fig. 1 shows a diagram describing the process.

The nucleus $A$, named TH nucleus, is a two-body system selected for its high probability for a cluster configuration $x \oplus s$. The beam energy is fixed such that $a+A$ interaction takes place above the $a-A$ Coulomb barrier. In this condition the TH nucleus breaks up in the nuclear field of $a$. The cluster $x$ interacts with $a$ inducing the two-body process of interest, while cluster $s$, the so-called spectator, flies away without interfering with the $x-a$ interaction. In a more detailed description it has to be taken into account that $x$ is a virtual particle, consequently its 


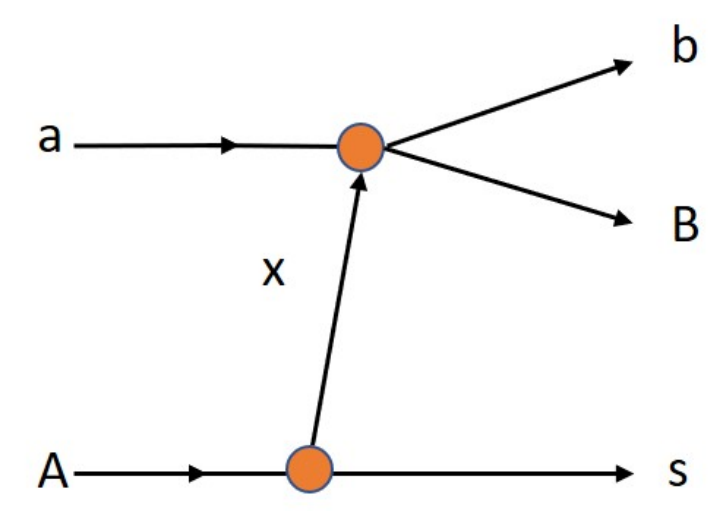

Figure 1: Diagram describing the TH reaction $a+A \rightarrow b+B+s$. The upper vertex describes the $x+a \rightarrow b+B$ two-body process of interest, while the lower one the virtual decaying of $\mathrm{A}$ as $\mathrm{x} \oplus \mathrm{s}$.

energy does not follow the mass-shell equation $E_{x} \neq p_{x}^{2} / 2 m_{x}$. For this reason the $x+A \rightarrow b+B$ two-body cross section extracted from the TH reaction is Half-Off-Energy-Shell, because at the same time it is true that all the outcoming particles can be detected.

Saying that cluster s remains spectator to the two-body process means that in the final state it should keep the same momentum it had inside A. If we take into account TH nuclei with $l=0$ inter-cluster motion, the QF mechanism gives the maximum contribution when the relative $x-s$ momentum $p_{x s}$ is zero. This condition implies that the $x-s$ relative distance is very large justifying the spectator role of the $s$ cluster.

The THM is an indirect method that can be applied to get the cross section for non-resonant as well as resonant binary processes. The relative theory of THM is extensively described in [2], as an example. For the simplest non-resonant case we can use many theoretical approaches, but describing both entrance and exit channel in terms of plane waves and using impulse approximation the three-body cross section (measured with the TH experiments) can be easily factorized as:

$$
\frac{d^{3} \sigma}{d E_{c m} d \Omega_{b} d \Omega_{B}} \propto \operatorname{KF}\left|\phi\left(p_{x s}\right)\right|^{2}\left(\frac{d \sigma_{x a \rightarrow b B}}{d \Omega}\right)^{H O E S},
$$

where the first term $K F$ is the kinematical factor, function of the masses, momenta and angles of the outgoing particles, representing the phase space, while $\left|\phi\left(p_{x s}\right)\right|^{2}$ is the momentum distribution given by the square modulus of the Fourier transform of the radial wave function describing the $x-s$ relative motion inside nucleus $A$. Typically ${ }^{2} \mathrm{H},{ }^{3} \mathrm{He}$ and ${ }^{6} \mathrm{Li}$ are used as TH nucleus, respectively as proton/neutron, deuteron, $\alpha$ virtual sources, where $\left|\phi\left(p_{x s}\right)\right|^{2}$ is described in terms of Húlten, Hänkel and Eckart functions respectively.

Finally, the last factor is the differential cross section for the two-body process in the center of mass $(\mathrm{cm})$ system. Energy in the $\mathrm{cm}$ system $E_{c m}$ is calculated following the post-collision prescription [2]:

$$
E_{c m}=E_{b B}-Q_{2 b},
$$

where $E_{b B}$ is the $b-B$ relative energy and $Q_{2 b}$ is the Q-value of the two-body subprocess. The superscript HOES in eq. 1 underlines that $x+a \rightarrow b+B$ is Half-Off-Energy-Shell, due to the virtuality of the transferred particle $x$. 


\section{THM for nuclear astrophysics}

The possibility to extract the cross section of a binary process at sub-Coulomb energies settles THM as a powerful experimental technique to study nuclear reactions of astrophysical interest. In astrophysical environments, nuclear reactions take place at very low energy of the order of keV's. For reactions between charged particles, this means that their interaction takes place at energies well below their Coulomb barrier, that is of the order of MeV's. Consequently, the cross section $\sigma_{b}(E)$ drops exponentially with decreasing energy and reaches values of the order of $10^{-9}-10^{-12}$ barn (subscript $b$ is used to indicate the bare nuclei cross section). In these conditions, the experimental evaluation of the cross section is severely hindered and in some cases even beyond the technical possibilities. Extrapolation from data at higher energies represents a possible solution but the exponential decreasing of the cross section could be source of important systematic errors. The astrophysical $S(E)$ factor

$$
S(E)=E \sigma_{b}(E) \exp (2 \pi \eta)
$$

is introduced to better look at the data available in literature with a much weaker energy dependence, since the Gamow factor $\exp (2 \pi \eta)$ compensates for the exponential decreasing of $\sigma_{b}(E)$ (here $\eta$ is the Sommerfeld parameter). Even if the introduction of $S(E)$ facilitates the extrapolation procedure, uncertainties can arise due to the contribution of tails of possible sub-threshold resonances [3]. The experimental studies that have been able to perform measurements within the energy range of astrophysical interest have highlighted an unexpected effect, named electron screening, due to the atomic electrons surrounding both projectile and target nuclei [4], which screens the Coulomb barrier and produces, at ultra-low energies, an enhancement of the cross section with respect to the bare nucleus case. The enhancement factor $f_{l a b}(E)$ is given by the following equation:

$$
f_{l a b}(E)=\frac{\sigma_{s}(E)}{\sigma_{b}(E)} \sim \exp \left[\pi \eta\left(\frac{U_{e}}{E}\right)\right],
$$

where $U_{e}$ is the electron screening potential (subscript $s$ refers to screened cross section). Since the plasma condition of the matter within stars leads to a different value of the enhancement factor with respect to the laboratory case, the bare nucleus cross section $\sigma_{b}(E)$ is the required experimental value. The only way to get $\sigma_{b}(E)$ is still with an extrapolation procedure even when experimental data are available down to the astrophysical energy range. In this framework many indirect methods have been developed with the aim to extract the bare nucleus cross section for reactions of astrophysical interest, avoiding experimental problems due to Coulomb suppression and electron screening effect and among them an important role is played by the Trojan Horse Method [2]. As an example of its impact, a recent outstanding study on Carbon burning in stars has been published [5].

\section{The $d+d$ reactions studied via the THM}

A great experimental effort has been devoted to the study of $\mathrm{p}-{ }^{3} \mathrm{H}$ and $\mathrm{n}-{ }^{3} \mathrm{He}$ channels of the $d+d$ reaction, because of their crucial role at low energies for astrophysics and for applied physics. Indeed, ${ }^{2} \mathrm{H}(\mathrm{d}, \mathrm{p})^{3} \mathrm{H}$ and ${ }^{2} \mathrm{H}(\mathrm{d}, \mathrm{n}){ }^{3} \mathrm{He}$ reactions are in the network of the twelve most influent Standard Big Bang Nucleosynthesis (SBBN) reactions, being strongly influential for all the primordial abundances [6], and are among the deuterium burning channels in the Pre Main Sequence (PMS) stars [3]. Moreover, these reactions are among the few possible cases to be used in the future fusion power plants [7] [8]. About the former topic, the energy range 
of interest spans from 0 to $1.5 \mathrm{MeV}$ (in order to have an accurate reaction rate calculation), while in the latter from 0 to $30 \mathrm{keV}$.

The first attempt of the THM application to $d+d$ interaction has focused on the ${ }^{2} \mathrm{H}(\mathrm{d}, \mathrm{p})^{3} \mathrm{H}$ channel. The selected $\mathrm{TH}$ reaction was ${ }^{2} \mathrm{H}\left({ }^{6} \mathrm{Li}, \mathrm{pt}\right){ }^{4} \mathrm{He}$. The experiment was performed using a $14 \mathrm{MeV}^{6} \mathrm{Li}$ beam on a $\mathrm{CD}_{2}$ target (thickness $174 \mu \mathrm{g} / \mathrm{cm}^{2}$ ). In this case, the TH nucleus was ${ }^{6} \mathrm{Li}$, where the participant cluster was a deuteron, whereas the $\alpha$ particle was the spectator one. Details of this experiment can be found in [9], where it is shown how results coming from this run were affected by the presence of a sequential decay (SD) reaction mechanism. For this reason, a substantial cut on available data was required, leading to a $S(E)$ poor in resolution.

That is why other two experimental runs were necessary to improve the result, using ${ }^{3} \mathrm{He}$ as TH nucleus. The THM was applied to ${ }^{2} \mathrm{H}\left({ }^{3} \mathrm{He}, \mathrm{p}^{3} \mathrm{H}\right) \mathrm{H}$ and ${ }^{2} \mathrm{H}\left({ }^{3} \mathrm{He}, \mathrm{n}^{3} \mathrm{He}\right) \mathrm{H}$ three-body processes induced by a ${ }^{3} \mathrm{He}$ beam (with 17 and $18 \mathrm{MeV}$ energies) impinging on $\mathrm{CD}_{2}$ target (with thickness $150 \mu \mathrm{g} / \mathrm{cm}^{2}$ ). In order to avoid the SD contamination, ${ }^{3} \mathrm{He}$ (whose internal structure has been deeply studied theoretically [10]) was used as TH nucleus, as a source of virtual deuteron, the proton being the spectator particle. Details for these runs can be found in [11].

The first run was devoted exclusively to the $\mathrm{p}-{ }^{3} \mathrm{H}$ measurement (see [12]), looking for an improvement of statistics and, as further discussed, to test the effects of a different TH nucleus. With the second run, we reached the desired S(E) for both channels, and particularly in the case of $n-{ }^{3} \mathrm{He}$ channel it has been very advantageous to detect the spectator proton, to get over the troubles related to the neutrons detection. This very useful spectator detection is possible because it is necessary to detect just two of the three outcoming particles in any TH experiment, being the third particle energy and angle reconstructed by energy and momentum conservation laws.

Using a more sophisticated analysis, that made use of MPWBA (Modified Plane Wave Born Approximation) [13] [14], results have a substantial improvement with respect to the previous run. The desired two-body cross sections can be considered as made of two non-resonant components for the $l=0,1$ partial waves. Their energy dependence is described by their penetrability factors [15], so that, writing the two channels $p-{ }^{3} \mathrm{H}$ and $n-{ }^{3} \mathrm{He}$ as $\mathrm{C}+\mathrm{c}$, one obtains:

$$
\frac{d \sigma}{d E d \Omega}_{(d+d \rightarrow C+c)}=\frac{1}{E_{d d}} \sum_{l=0,1} C_{l} P_{l}^{2} k_{d d} R T_{l}\left(k_{d d} R\right),
$$

where the relative energy $E_{d d}$ is connected to the momentum $k_{d d}$, to the reduced mass $\mu_{d d}$ and to the binding energy $B_{d p}$ of ${ }^{3} \mathrm{He}$ by the relation $E_{d d}=\frac{\hbar k_{d d}^{2}}{2 \mu_{d d}}-B_{d p}$. Moreover, $C_{l}$ are the scaling factors and $P_{l}$ the penetrability factors related to the two partial waves considered.

To apply this MPWBA approach has been necessary to know the values of the scaling factors $C_{l=0,1}$, thus eq. 5 entered in eq. 1 to fit the experimental three-body coincidence yield, using $C_{l}$ and the channel radius $\mathrm{R}$ as free parameters, and values obtained are reported in [11].

Besides widely energy extended S(E) (from 0 to $1.5 \mathrm{MeV}$ ), other results obtained have been significative, meaning a very precise reaction rate evaluation throughout the region of interest for astrophysical scenarios (with less than 5\% error), differing up to $20 \%$ with respect to previous calculations [15] [16] [17], whose effects are considered in all the SBBN model with proper calculations [18].

Furthermore, the result of the electron screening potential estimate is significant. As mentioned before, nuclear reactions performed in the laboratory are affected by a different screening than in plasma [19]. Morevover, for the present case it is particularly important to measure the bare nucleus cross section to avoid critical errors in the fusion power prototype plants. That is why it is not sufficient, as usual, a simple extrapolation of available unscreened data at higher energy, due to the uncertainty that this procedure implies. A careful analysis of these results is summarized in [11]. 
It is also worth noticing the nice concordance of results in [9] and [11] for the ${ }^{2} \mathrm{H}(\mathrm{d}, \mathrm{p})^{3} \mathrm{H}$ $\mathrm{S}(\mathrm{E})$, recalling here that the first case has been obtained using ${ }^{6} \mathrm{Li}$ and the second using ${ }^{3} \mathrm{He}$ as deuteron inducer. The comparison, shown in fig. 2, clearly makes evidence of the method independence from the TH-nucleus used, as claimed in [20], but it is also outstanding the agreement with polynomial fits by [17] and [16], as well as the ab initio calculation from [21], adding a further validity test for the method itself.

This result also resembles what obtained for the cases of ${ }^{7} \operatorname{Li}(\mathrm{p}, \alpha) \alpha$ and ${ }^{6} \operatorname{Li}(\mathrm{d}, \alpha) \alpha$, where similarly different TH nuclei have been used obtaining the same trend for S(E) [22] and definitely in [23].

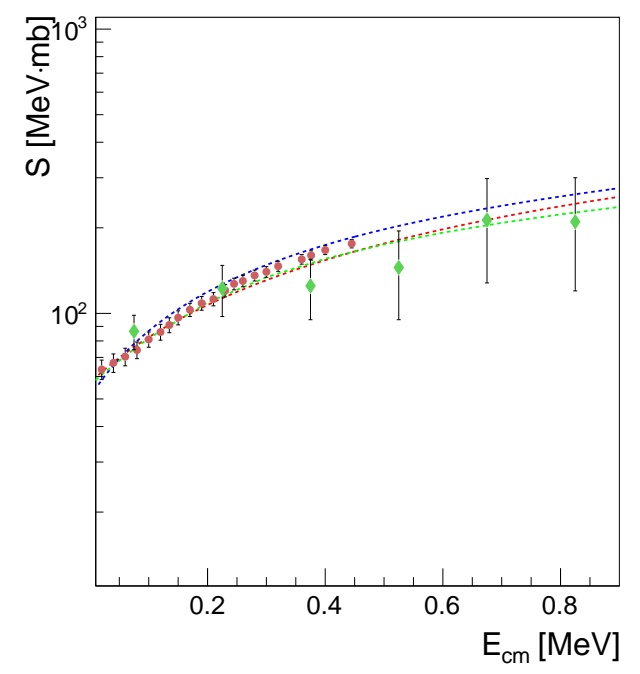

Figure 2: Bare nucleus TH S(E) for $\mathrm{p}{ }^{3} \mathrm{H}$ channel obtained by the ${ }^{6} \mathrm{Li}$ break-up [9] (as green diamonds) and by the ${ }^{3} \mathrm{He}$ break-up [11] (as red dots). The red and blue lines are the polynomial fit by [17] and [16], while the green line is the $a b$ initio calculation by [21].

\section{Conclusions}

Considering what mentioned in the previous section and that in recent years THM has been extended to neutron induced and radioactive beams induced reactions, succesfully in both cases (as examples [24], [25] and references therein), it has been finally proved that this method is a valid help for nuclear measurements also in such complex experimental contexts.

\section{References}

[1] G. Baur, Breakup reactions as an indirect method to investigate low-energy chargedparticle reactions relevant for nuclear astrophysics, Phys. Lett. B 178, 135 (1986), doi:10.1016/0370-2693(86)91483-8.

[2] C. Spitaleri et al., The ${ }^{11} B\left(p, \alpha_{0}\right)^{8}$ Be reaction at sub-Coulomb energies via the Trojan-horse method, Phys. Rev. C 69, 055806 (2004), doi:10.1103/PhysRevC.69.055806. 
[3] C. Rolfs and W.S. Rodney, Cauldrons in the Cosmos, Chicago, IL: Univ. Chicago Press (1988).

[4] H. J. Assenbaum, K. Langanke and C. Rolfs, Effects of electron screening on low-energy fusion cross sections, Z. Phys. A - Atomic Nuclei 327, 461 (1987), doi:10.1007/BF01289572.

[5] A. Tumino et al., An increase in the ${ }^{12} \mathrm{C}+{ }^{12} \mathrm{C}$ fusion rate from resonances at astrophysical energies, Nature 557, 687 (2018), doi:10.1038/s41586-018-0149-4.

[6] G. Steigman, Primordial nucleosynthesis: successes and challenges, Int. J. Mod. Phys. E 15, 1 (2006), doi:10.1142/S0218301306004028.

[7] J. Lindl, Development of the indirect-drive approach to inertial confinement fusion and the target physics basis for ignition and gain, Phys. Plasmas 2, 3933 (1995), doi:10.1063/1.871025.

[8] C. Yamanaka, Inertial confinement fusion: The quest for ignition and energy gain using indirect drive, Nucl. Fusion 39, 825 (1999), doi:10.1088/0029-5515/39/6/702.

[9] A. Rinollo et al., Measurement of cross section and astrophysical factor of the $d(d, p) t$ reaction using the Trojan Horse Method, Nucl. Phys. A 758, 146 (2005), doi:10.1016/j.nuclphysa.2005.05.030.

[10] A. Kievsky, S. Rosati, M. Viviani, L. E. Marcucci and L. Girlanda, A high-precision variational approach to three- and four-nucleon bound and zero-energy scattering states, J. Phys. G: Nucl. Part. Phys. 35, 063101 (2008), doi:10.1088/0954-3899/35/6/063101.

[11] A. Tumino et al., New determination of the ${ }^{2} H(d, p)^{3} H$ and ${ }^{2} H(d, n)^{3} H e$ reaction rates at astrophysical energies, Astrophys. J. 785, 96 (2014), doi:10.1088/0004-637X/785/2/96.

[12] R. Spartaá et al., Indirect approach to the ${ }^{2} H(d, p)^{3} H$ reaction study, AIP Conf. Proc. 1304, 420 (2010), doi:10.1063/1.3527238.

[13] S. Typel and G. Baur, Theory of the Trojan-Horse method, Ann. Phys. 305, 228 (2003), doi:10.1016/S0003-4916(03)00060-5.

[14] S. Typel and H. H. Wolter, Extraction of astrophysical cross sectionsin the Trojan-Horse method, Few-Body Syst. 29, 75 (2000), doi:10.1007/s006010070010.

[15] P. Descouvemont, A. Adahchour, C. Angulo, A. Coc and E. Vangioni-Flam, Compilation and R-matrix analysis of Big Bang nuclear reaction rates, At. Data Nucl. Data Tables 88, 203 (2004), doi:10.1016/j.adt.2004.08.001.

[16] R. H. Cyburt, Primordial nucleosynthesis for the new cosmology: Determining uncertainties and examining concordance, Phys. Rev. D 70, 023505 (2004), doi:10.1103/PhysRevD.70.023505.

[17] C. Angulo et al., A compilation of charged-particle induced thermonuclear reaction rates, Nucl. Phys. A 656, 3 (1999), doi:10.1016/S0375-9474(99)00030-5.

[18] R. G. Pizzone, R. Spartá, C. A. Bertulani, C. Spitaleri, M. La Cognata, J. Lalmansingh, L. Lamia, A. Mukhamedzhanov and A. Tumino, Big Bang nucleosynthesis revisited via Trojan Horse method measurements, Astrophys. J. 786, 112 (2014), doi:10.1088/0004$637 \mathrm{X} / 786 / 2 / 112$. 
[19] G. Shaviv and N. J. Shaviv, Is there a dynamic effect in the screening of nuclear reactions in stellar plasmas?, Astrophys. J. 529, 1054 (2000), doi:10.1086/308290.

[20] R. G. Pizzone et al., Updated evidence of the Trojan horse particle invariance for the ${ }^{2} H(d, p){ }^{3} H$ reaction, Phys. Rev. C 87, 025805 (2013), doi:10.1103/PhysRevC.87.025805.

[21] K. Arai, S. Aoyama, Y. Suzuki, P. Descouvemont and D. Baye, Tensor force manifestations in ab initio study of the ${ }^{2} \mathrm{H}(\mathrm{d}, \gamma)^{4} \mathrm{He},{ }^{2} \mathrm{H}(\mathrm{d}, \mathrm{p})^{3} \mathrm{H}$, and ${ }^{2} \mathrm{H}(\mathrm{d}, n)^{3} \mathrm{He}$ reactions, J. Phys.: Conf. Ser. 436, 012024 (2013), doi:10.1088/1742-6596/436/1/012024.

[22] R. G. Pizzone et al., Trojan horse particle invariance studied with the ${ }^{6} \mathrm{Li}(\mathrm{d}, \alpha){ }^{4} \mathrm{He}$ and ${ }^{7} \mathrm{Li}(p, \alpha){ }^{4} \mathrm{He}$ reactions, Phys. Rev. C 83, 045801 (2011), doi:10.1103/PhysRevC.83.045801.

[23] R. G. Pizzone et al., Trojan Horse particle invariance: An extensive study, Few-Body Syst. 55, 1001 (2014), doi:10.1007/s00601-014-0829-z.

[24] L. Lamia et al., Cross-section measurement of the cosmologically relevant ${ }^{7} \mathrm{Be}(n, \alpha){ }^{4} \mathrm{He}$ reaction over a broad energy range in a single experiment, Astrophys. J. 879, 23 (2019), doi:10.3847/1538-4357/ab2234.

[25] G. L. Guardo et al., Assessing the near threshold cross section of the ${ }^{17} O(n, \alpha){ }^{14} C$ reaction by means of the Trojan horse method, Phys. Rev. C 95, 025807 (2017), doi:10.1103/PhysRevC.95.025807. 